\title{
Moderow, Hans-Martin, Volksschule zwischen Staat und Kirche. Das Beispiel Sachsen im 18. und 19. Jahrhundert
}

Jean-Luc Le Cam

\section{OpenEdition}

Journals

Édition électronique

URL : http://journals.openedition.org/ifha/1608

DOI : $10.4000 /$ ifha. 1608

ISSN : 2198-8943

Éditeur

IFRA - Institut franco-allemand (sciences historiques et sociales)

Référence électronique

Jean-Luc Le Cam, « Moderow, Hans-Martin, Volksschule zwischen Staat und Kirche. Das Beispiel Sachsen im 18. und 19. Jahrhundert », Revue de l'IFHA [En ligne], Date de recension, mis en ligne le 01 janvier 2008, consulté le 22 septembre 2020. URL : http://journals.openedition.org/ifha/1608 ; DOI : https:// doi.org/10.4000/ifha. 1608

Ce document a été généré automatiquement le 22 septembre 2020.

(CIFHA 


\title{
Moderow, Hans-Martin, Volksschule zwischen Staat und Kirche. Das Beispiel Sachsen im 18. und 19. Jahrhundert
}

\author{
Jean-Luc Le Cam
}

1 Lorsque Victor Cousin entreprit son voyage outre-Rhin afin de rédiger son Rapport sur l'état de l'instruction publique dans quelques pays d'Allemagne (1832), il avait comme but principal la Prusse, mais il voulut aussi faire étape dans le Royaume de Saxe dont l'école primaire passait déjà pour un modèle du genre. De fait, l'école saxonne a conservé pendant tout le XIXe s. et le début du XXe s. une image flatteuse. C'est pour vérifier avec les moyens de la recherche actuelle les réalités que recouvrait cette réputation que l'auteur a entrepris cette thèse sous la direction d'Ulrich von Hehl à l'Université de Leipzig dans le cadre du Sonderforschungsbereich « Regionenbezogene Identifikationsprozesse » (« Processus d'identifications régionaux », dir. Heinz-Werner Wollersheim). Elle se donne pour but de faire une revue sur le long terme du développement du système scolaire primaire de la Saxe de la fin du XVIIIe s. jusqu'aux années 1870 (les indications chronologiques données dans le titre du livre apparaissent un peu trop extensives). L'auteur entend ainsi contribuer, après d'autres recherches régionales, à élargir la perspective trop partielle et focalisée sur la Prusse de l'histoire de l'école allemande de cette période. Sa problématique est des plus classique : il s'agit d'étudier la politique scolaire dans ses dimensions constitutionnelles, administratives et institutionnelles tout en prenant en compte « la problématique de la nouvelle historiographie de l'éducation » (p. 40). En fait l'auteur est historien et non pas issu des sciences de l'éducation, de sorte qu'il accorde moins d'intérêt aux aspects proprement pédagogiques. Ses sources principales sont essentiellement les archives des autorités décisionnelles, ici le Kultusministerium de Saxe et le Landtag, et des autorités intermédiaires de contrôle. Les sources qui reflètent un peu mieux les positions des acteurs de base se réduisent aux écrits et à la presse des associations d'enseignants.

2 Le plan est donc très classiquement chronologique : six chapitres présentent les phases successives du développement du système et de la politique scolaire. D’abord la période 
autour de 1800, qui voit les premières réformes dans le domaine de l'école primaire, avec une accélération dans les années 1800-1816 et qui permet aussi de dresser un tableau de départ. Puis (chapitre 2) la période des grandes réformes de l'État (1831) et la loi scolaire de 1835 dans le contexte du libéralisme et du néoluthéranisme : le projet de loi préparé par Gottlob Leberecht Schulze établit une réglementation globale de l'école primaire qui est considérée comme un modèle en Allemagne. Cette législation ne se conçoit que dans le cadre de la grande réforme de l'État en Saxe qui aboutit entre autres à la création d'un ministère du culte et de l'instruction publique en 1831 (Kultusministerium) et à l'unification et à la rationalisation de l'administration scolaire sur le terrain. Le chapitre 3 traite des " tempêtes » du Vormärz, c'est-à-dire des premiers assauts des novateurs, pédagogues partisans de la psychologie, adeptes d'une critique " éclairée " vis-à-vis des confessions (mouvement des Lichtfreunde et des Catholiques allemands) et libéraux contre le système dont ils veulent la réforme, ce qu'ils manifestent au moyen de pétitions. Mais jusqu'en 1848, c'est le système scolaire secondaire qui mobilise pour l'essentiel la politique scolaire. La révolution de 1848-50 (chapitre 4) voit les enseignants rejoindre en grand nombre le parti démocratique et prendre parfois des positions radicales sans que leurs projets de réforme soient réalisés. Le temps de la Réaction (chapitre 5) n'a pas pour autant conduit selon l'auteur à des positions très répressives vis-à-vis des enseignants. Toutefois, on note de nombreuses mesures de détail dans le domaine de la discipline imposée aux maîtres et aux séminaristes, une réforme de la formation dans les séminaires et la nomination d'un personnel politique nettement conservateur et orthodoxe à la tête de l'administration scolaire. L'auteur les présente comme des concessions à l'assemblée conservatrice du Landtag destinées à obtenir en échange une amélioration salariale pour les maîtres. Dans les années 1860 commence (chapitre 6) une ère libérale qui réforme profondément le système mis en place en 1835 et aboutit à la législation de 1873 pour les écoles primaires, de 1876 pour les lycées, les Realschulen et les séminaires. Cette législation, qui a été âprement débattue et s'est imposée dans la lignée du Kulturkampf contre l'opposition de l'orthodoxie luthérienne, crée pour la première fois une surveillance de l'école détachée de l'Église et confiée à des hommes de l'art.

Un dernier chapitre est spécialement consacré à l'histoire des séminaires de formation des maîtres, ce qui conduit forcément à certaines redites, notamment sur le contexte politique. Après de premières tentatives au XVIIIe s., ce système de formation trouve son véritable départ dans le cadre de la législation de 1835 qui unifie son administration et son système d'examen. Dans les années 1840, la formation est homogénéisée à l'échelle du pays sur la base d'un cursus de 4 ans et est portée à 6 ans en 1873 par l'intégration des Proseminare (séminaires de premier cycle). L'ordonnance de 1859 est à la fois modernisatrice dans son effort de simplification et de concentration de l'enseignement et conservatrice dans sa volonté de former des enseignants bien disposés vis-à-vis de l'Église. La parenté avec les Regulative de Stiehl en Prusse est frappante même si les antécédents et le contexte sont différents. Les besoins en recrutement de maîtres conduisent à l'extension du système et à la possibilité pour les meilleurs séminaristes d'accéder à l'Université à partir de 1865 . L'auteur estime en conclusion du chapitre que ce système de formation des maîtres était en pointe par rapport au reste de l'Allemagne. D'une façon globale, l'efficience du système scolaire est attestée selon lui par les excellents taux d'alphabétisation (95\% en 1830 , presque $100 \%$ en 1874 ). 
4 Le mérite de ce travail est de reprendre avec beaucoup de sérieux et le recours à diverses sources la question de la politique scolaire. Le sujet méritait en effet de nouvelles recherches depuis l'ouvrage de Julius Richter (1930) qui restait dans la logique des Monumenta Paedagogica, celle d'une histoire de pédagogues. Cependant, c'est reprendre dans l'autre sens les œillères de ce type d'histoire que de s'enorgueillir en conclusion d'avoir permis de comprendre la logique propre du temps grâce au " regard désintéressé (car non pédagogique) de l'historien »; et méconnaître justement les apports de la nouvelle historiographie de l'éducation. D'autre part, et précisément d'un point de vue d'historien, on peut s'étonner de cette réduction de la perspective au champ politique et institutionnel alors qu'il y aurait tant à dire et à chercher sur les aspects économiques, sociaux et culturels qui ont marqué le développement de l'école primaire en Saxe entre les années 1770 et 1870.

5 Jean-Luc Le Cam (Université de Brest) 